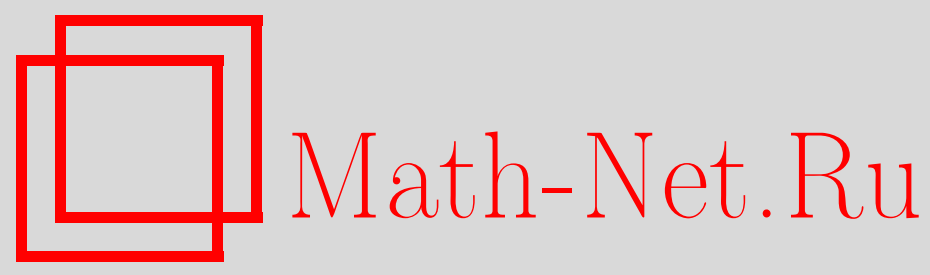

В. С. Балаганский, Необходимые условия дифференцируемости функции расстояния, Матем. заметки, 2002, том 72, выпуск 6, 815-820

DOI: https://doi.org/10.4213/mzm468

Использование Общероссийского математического портала Math-Net.Ru подразумевает, что вы прочитали и согласны с пользовательским соглашением http://www.mathnet.ru/rus/agreement

Параметры загрузки:

IP : 54.164 .48 .24

26 апреля 2023 г., 18:18:09

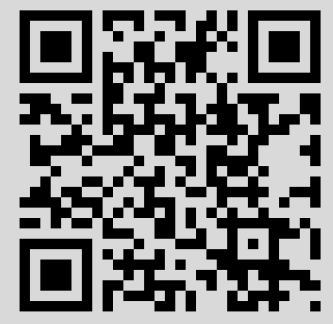


УДК 517.5

\section{НЕОБХОДИМЫЕ УСЛОВИЯ \\ ДИФФЕРЕНЦИРУЕМОСТИ ФУНКЦИИ РАССТОЯНИЯ}

\section{В. С. Балаганский}

Рассматриваются необходимые условия дифференцируемости по Гато функции расстояния до множества. Получен ряд характеризационных результатов.

Библиография: 11 названий.

В работе рассматриваются необходимые условия дифференцируемости по Гато функции расстояния от точки до множества. Ранее дифференцируемостью функции расстояния занимались в работах [1]-[10]. Указаны необходимые условия, совпадающие с полученньми ранее достаточными; тем самым, улучшены результаты, имевшиеся в обзорной статье [8].

В работе приняты следующие обозначения:

$X$ - вещественное банахово пространство;

$\bar{A}, \partial A$ - соответственно замькание, граница множества $A \subset X$;

$\mathbb{B}(x, r)=\{z \in X: z x \leqslant r\}-$ шар в $X$;

$\mathbb{S}(x, r)=\{z \in X: z x=r\}-$ cфера;

$\mathbb{B}(Z), \mathbb{S}(Z)$ - единичные шар и сфера в $Z ;$

$x y=\|x-y\|$;

$x A=d(x, A)=\inf \{x y: y \in A\}-$ расстояние от $x$ до $A \subset X$;

$P_{M}: x \mapsto P_{M}(x)=\{y \in M: x y=x M\} ;$

$\delta S(M)=\delta S_{M}-$ множество $\delta$-солнечны последовательность $v_{n} \neq x, v_{n} \rightarrow x$, для которой

$$
\frac{d\left(v_{n}, M\right)-d(x, M)}{\left\|v_{n}-x\right\|} \rightarrow 1
$$

$\delta^{\prime} S_{M}$ - множество тех $x \in X \backslash M$, для которых существуют $h \in \mathbb{S}(X), t_{n} \rightarrow+0$ такие, что для $v_{n}=x+t_{n} h$ имеем (1);

$E(M)=\left\{x \in X: P_{M}(x) \neq \varnothing\right\}$-множество точек существования элемента наилучшего приближения;

$G_{M}$-множество тех $x \in X \backslash M$, где $d(\cdot, M)$ имеет производную Гато $d^{\prime}(x, M)=f \in X^{*}$, так что для всех $h \in X$

$$
f(h)=\lim _{t \rightarrow 0} \frac{d(x+t h, M)-d(x, M)}{t} ;
$$

Работа вьполнена при поддержке программы “Ведущие научные школы”, грант № 00-15-96035. 
$G_{M}^{1}=\left\{x \in G_{M}:\|d(x, M)\|=1\right\} ;$

$\Gamma_{M}^{1}-$ множество точек $x \in X \backslash M$ таких, что для некоторого $h \in \mathbb{S}(X)$ предел (2) равен 1 при $t \rightarrow+0$;

$T(M)=E(M) \cap U(M)$;

$T_{M}^{\prime}=\{x \in X: \operatorname{diam}(\mathbb{B}(x, x M+\delta) \cap M) \rightarrow 0$ при $\delta \rightarrow+0\} ;$

$\tau A C(M)$ - множество точек аппроксимативной $\tau$-компактности, т.е. тех $x \in X \backslash M$, для которых всякая минимизирующая последовательность $y_{n}$ (т.е. $y_{n} \in M, x y_{n} \rightarrow x M$ ) содержит подпоследовательность, $\tau$-сходящуюся к элементу из $M$ (зависящему от подпоследовательности);

в этой работе множество, подлежащее изучению, всегда обозначается через $M$; при этом предполагается, что $M=\bar{M} \neq X$;

$\mathscr{F}=\mathscr{F}(X)-$ семейство всех таких $M$;

$\mathscr{F}_{\text {сс }}(X)$ - семейство всех тех $M \in \mathscr{F}(X)$, для которых $X \backslash M$ вьпукло;

через $\tau$ обозначается любая из топологий: $n$ (нормированная, или сильная; $n$ обычно опускается), $w$ (слабая), $w \omega$ (секвенциальная слабая), $w^{*}$ (слабая* сопряженного пространства);

$(R f)$ - класс рефлексивных пространств;

$(R)$ - класс строго вьпуклых пространств (т.е. таких, что единичная сфера $\mathbb{S}(X)$ не содержит отрезков);

$(S)$ - класс гладких пространств (в каждой точке сферы существует единственная опорная гиперплоскость);

$(\tau L U R)-\tau$-локально равномерно выпуклые пространства: если $x, x_{n} \in \mathbb{S}(X), \| x+$ $x_{n} \| \rightarrow 2$, то $x_{n} \stackrel{\tau}{\longrightarrow} x$;

$(F)$ - класс пространств с нормой, дифференцируемой по Фреше в каждой точке $x \in$ $\mathbb{S}(X)$

$(C D)$ - класс пространств, в которьх из условий $x, x_{n} \in \mathbb{S}(X), f \in \mathbb{S}\left(X^{*}\right), f(x)=1$, $f\left(x_{n}\right) \rightarrow 1$ вытекает, что $x_{n}$ имеет сходящуюся подпоследовательность;

$\left(w^{*} \omega C D^{*}\right)$ - класс сопряженных пространств $X^{*}$, в которых из условий $x \in \mathbb{S}(X)$, $f_{n} \in \mathbb{S}\left(X^{*}\right), f_{n}(x) \rightarrow 1$ следует, что существует $w^{*}$-сходящаяся подпоследовательность $f_{n_{k}}$; в частности, $X^{*} \in\left(w^{*} \omega C D^{*}\right)$, если $X$ - рефлексивное, сепарабельное или гладкое банахово пространство.

Нам потребуются следующие утверждения.

ПРЕДЛОЖЕНИЕ А (см. [8, предложение 1.2]). Для любого $M \in \mathscr{F}(X)$ справедливь соотношения

$$
F_{M} \subset G_{M}^{1} \subset \delta S_{M}, \quad G_{M} \cap E(M)=G_{M}^{1} \cap E(M),
$$

a если $X \in(R f)$, mo $G_{M}^{1} \subset \Gamma_{M}^{\prime}$.

Teоpema A [8, теорема 1.2]. Пусть $M \in \mathscr{F}(X)$. Тогда

a) если $X \in(L U R)$, mo

a') $\delta^{\prime} S_{M} \subset T_{M}^{\prime}$

$\left.\mathrm{a}^{\prime \prime}\right) \delta S_{M} \cap E(M) \subset T_{M}^{\prime}$

б) если $X \in(\tau L U R)$, mo $\delta^{\prime} S_{M} \cap E(M) \subset T(M) \cap \tau A C(M)$. 
Tеорема Б [8, с. 144]. Рассмотрим утверждения:

a) $X \in(w L U R)$;

б) $G_{M} \cap E(M) \subset T(M) \cap w A C(M)$ для всех $M \in \mathscr{F}(X)$ (вариант: для всех $\left.M \in \mathscr{F}_{\mathrm{cc}}(X)\right)$.

Тогда а) $\Longrightarrow$ б), а если $X \in(S)$, то б) $\Longrightarrow$ а).

Теорема 1. Пусть $X$ - банахово пространство такое, что $X^{*}$ удовлетворяет условию $\left(w^{*} \omega C D^{*}\right)$. Тогда если $X \notin(\tau L U R)$, то существуют $M \in \mathscr{F}_{\mathrm{cc}}(X)$ и точка $x \in\left(G_{M}^{1} \cap E(M)\right) \backslash(T(M) \cap \tau A C(M))$.

ДокАЗАТЕЛЬСтво. Если $X$ не является строго вьпуклым пространством, то найдутся точки $y, z \in \mathbb{S}(X)$ такие, что $\|y+z\|=2, y \neq z$. По теореме Хана-Банаха найдется функционал $g \in \mathbb{S}\left(X^{*}\right)$ такой, что $g(y+z)=\|g\| \cdot\|y+z\|=2$. Тогда для множества $M=\{x \in X: g(x) \geqslant 1\}$ имеем $x=0 \in\left(G_{M}^{1} \cap E(M)\right) \backslash T(M)$. Допустим, что $X \in(R) \backslash(\tau L U R)$, т.е. существуют $x, x_{n} \in \mathbb{S}(X),\left\|x+x_{n}\right\| \rightarrow 2$, и $x_{n}$ не является $\tau$-сходящейся к $x$ последовательностью. Возьмем $f_{n} \in \mathbb{S}\left(X^{*}\right), f_{n}\left(x+x_{n}\right)=\left\|x+x_{n}\right\| \rightarrow 2$. Имеем $f_{n}\left(x_{n}\right) \leqslant 1, f_{n}(x) \leqslant 1$; следовательно, $f_{n}(x) \rightarrow 1$. В силу условия $\left(w^{*} \omega C D^{*}\right)$ считаем, что $f_{n} w^{*}$-сходится к $f_{0} \in \mathbb{B}\left(X^{*}\right)$. Так как $f_{n}(x) \rightarrow 1, x \in \mathbb{S}(X)$, то $f_{0}(x)=$ $1=\left\|f_{0}\right\|$. Пусть

$$
\begin{gathered}
M_{n}=\left\{z \in X: f_{n}(z) \geqslant 2+\frac{1}{n}\right\} \quad \text { при } n=1,2, \ldots, \\
M_{0}=\left\{z \in X: f_{0}(z) \geqslant 2\right\}, \quad M=\bigcup_{n=0}^{\infty} M_{n} .
\end{gathered}
$$

В силу $X \in(R)$ получаем, что $M_{n} \neq M_{0}$ при $n \neq 0$.

Докажем, что $M \in \mathscr{F}_{\text {сс }}(X)$. Вьпуклость $X \backslash M=\bigcap_{n=0}^{\infty}\left(X \backslash M_{n}\right)$ очевидна. Если $y_{n} \in M, y_{n} \rightarrow y$, то найдется подпоследовательность $k_{n}$ такая, что $y_{n} \in M_{k_{n}}$, $y M_{k_{n}} \rightarrow 0$. Если $y \in M_{k_{n}}$ при каком-нибудь $k_{n}$, то $y \in M$; если $y \notin M_{n}$ ни при каком номере $n$, то $y M_{k_{n}}=2+1 / k_{n}-f_{k_{n}}(y) \rightarrow 0$. Так как $f_{n} \stackrel{w^{*}}{\longrightarrow} f_{0}$, то $f_{0}(y)=2$ и $y \in M_{0} \subset M$. Тогда $M$ замкнуто, $M \in \mathscr{F}_{\text {cс }}(X)$.

Имеем $\left\|f_{n}\right\|=1, n=0,1,2, \ldots$ Тогда $\operatorname{int} \mathbb{B}(0,2) \subset X \backslash M_{n}$ при любом $n=0,1,2, \ldots$; следовательно, $d(0, M)=2$.

Имеем

$$
\begin{aligned}
x M & =\inf \left\{x M_{n}: n=0,1,2, \ldots\right\}=\min \left\{x M_{0}, \inf \left\{2+\frac{1}{n}-f_{n}(x): n=1,2, \ldots\right\}\right\} \\
& =\min \left\{1, \inf \left\{2+\frac{1}{n}-f_{n}(x): n=1,2, \ldots\right\}\right\}=1=x M_{0}
\end{aligned}
$$

следовательно, $x M=1<x M_{n}$ при любом $n=1,2, \ldots$ Тогда $2 x \in P_{M}(x), x \in E(M)$. Докажем, что для любого $y \in \mathbb{S}(X)$

$$
\lim _{t \rightarrow 0} \frac{\varphi(x+t y)-\varphi(x)}{t}=-f_{0}(y)
$$


Пусть $\lambda_{k}>0, \lambda_{k} \rightarrow 0, z_{k}=x+\lambda_{k} \cdot h$. Можно считать, что $z_{k} \notin M$. Тогда $\varphi\left(z_{k}\right)=\inf \left\{z_{k} M_{n}: n=0,1, \ldots\right\}=\min \left\{2-f_{0}\left(z_{k}\right), \inf \left\{2+\frac{1}{n}-f_{n}\left(z_{k}\right): n=1,2, \ldots\right\}\right\}$. Выберем последовательность $y_{k} \in M$ такую, что $z_{k} y_{k} \leqslant z_{k} M+\lambda_{k} / k$. Тогда $x y_{k} \rightarrow x M$. По построению для каждого номера $k$ найдется неотрицательное целое число $n_{k}$ такое, что $y_{k} \in M_{n_{k}}$. Если последовательность $n_{k}$ содержит стационарную подпоследовательность $n_{0}$, то получаем $\varphi(x)=d\left(x, M_{n_{0}}\right)=2+1 / n_{0}-f_{n_{0}}(x)>1$ для $n_{0}>0$; следовательно, $n_{0}=0, \varphi\left(z_{k}\right)=d\left(z_{k}, M_{0}\right)$ и для этой подпоследовательности равенство (3) установлено. Без потери общности можно считать, что $n_{k} \rightarrow \infty$ и предел

существует.

$$
\lim _{k \rightarrow \infty} \frac{\varphi\left(z_{k}\right)-\varphi(x)}{\lambda_{k}}
$$

Имеем

$$
\begin{aligned}
\lim _{k \rightarrow \infty} \frac{\varphi\left(z_{k}\right)-\varphi(x)}{\lambda_{k}} & =\lim _{k \rightarrow \infty} \frac{z_{k} y_{k}-\varphi(x)}{\lambda_{k}} \geqslant \underset{k \rightarrow \infty}{\lim _{k \rightarrow \infty}} \frac{z_{k} M_{n_{k}}-\varphi(x)}{\lambda_{k}} \\
& =\varliminf_{k \rightarrow \infty} \frac{2+1 / n_{k}-f_{n_{k}}\left(z_{k}\right)-\varphi(x)}{\lambda_{k}} \\
& =\underset{k \rightarrow \infty}{\frac{\lim }{k \rightarrow \infty}} \\
& \geqslant \underset{k \rightarrow \infty}{\lim _{k \rightarrow \infty}}-f_{n_{k}}(h)=-f_{0}(h) .
\end{aligned}
$$

Имеем $f_{n}(x) \rightarrow 1$; следовательно, для каждого номера $k$ найдется номер $i_{k} \geqslant k$ такой, что $\left(1+1 / i_{k}-f_{i_{k}}(x)\right) / \lambda_{k} \leqslant 1 / k$. Тогда получаем

$$
\begin{aligned}
\varlimsup_{k \rightarrow \infty} \frac{\varphi\left(z_{k}\right)-\varphi(x)}{\lambda_{k}} & \leqslant \varlimsup_{k \rightarrow \infty} \frac{z_{k} M_{i_{k}}-\varphi(x)}{\lambda_{k}}=\varlimsup_{k \rightarrow \infty} \frac{2+1 / i_{k}-f_{i_{k}}\left(z_{k}\right)-\varphi(x)}{\lambda_{k}} \\
& =\varlimsup_{k \rightarrow \infty} \frac{2+1 / i_{k}-f_{i_{k}}\left(\lambda_{k} h\right)-f_{i_{k}}(x)-\varphi(x)}{\lambda_{k}} \\
& =\varlimsup_{k \rightarrow \infty} f_{i_{k}}(h)=-f_{0}(h) .
\end{aligned}
$$

В силу произвольности $h \in \mathbb{S}(X)$ равенство (3) доказано, и справедливо $\varphi^{\prime}(x)=-f_{0}$, $\left\|\varphi^{\prime}(x)\right\|=\left\|f_{0}\right\|=1$.

Докажем, что $x \notin \tau A C(M) \cap T(M)$. Рассмотрим последовательность

$$
z_{n}=\left(2+\frac{1}{n}\right) \cdot \frac{x+x_{n}}{\left\|x+x_{n}\right\|} .
$$

Имеем $f_{n}\left(z_{n}\right)=2+1 / n$; следовательно, $z_{n} \in M_{n} \subset M$. Далее,

$$
\begin{aligned}
\lim _{n \rightarrow \infty}\left\|x-z_{n}\right\| & =\lim _{n \rightarrow \infty}\left\|x-\left(2+\frac{1}{n}\right) \cdot \frac{x+x_{n}}{\left\|x+x_{n}\right\|}\right\|=\lim _{n \rightarrow \infty}\left\|x-2 \cdot \frac{x+x_{n}}{\left\|x+x_{n}\right\|}\right\| \\
& =\lim _{n \rightarrow \infty}\left\|x-\left(x+x_{n}\right)\right\|=\lim _{n \rightarrow \infty}\left\|x_{n}\right\|=1=x M .
\end{aligned}
$$

Последовательность $x_{n}$ не является $\tau$-сходящейся к $x$ последовательностью; следовательно, последовательность $z_{n}$ не $\tau$-сходится к $2 x \in P_{M}(x)$.

С помощью теоремы 1 мы можем дополнить теорему Б следующим образом. 
СледСтвиЕ 1. Пусть $X$ - сепарабельное банахово пространство, $X^{*} \in$ $\left(w^{*} \omega C D^{*}\right)$. Следуюшие утверждения әквивалентны:

a) $X \in(\tau L U R)$;

б) $G_{M} \cap E(M) \subset T(M) \cap \tau A C(M)$ для всех $M \in \mathscr{F}(X)$;

в) $G_{M} \cap E(M) \subset T(M) \cap \tau A C(M)$ для всех $M \in \mathscr{F}_{\mathrm{Fc}}(X)$.

ДокАЗАтЕльСтво. Импликация а) $\Longrightarrow$ б). По предложению А имеем $G_{M} \cap E(M)=$ $G_{M}^{1} \cap E(M), G_{M}^{1} \subset \delta S_{M}$, по теореме А $\delta S_{M} \cap E(M) \subset T(M) \cap \tau A C(M)$. Импликация б) $\Longrightarrow$ в) очевидна. Импликация в) $\Longrightarrow$ а) по теореме 1 .

В [8] доказано, что в $X \in(w L U R)$ для $M \in \mathscr{F}(X)$ справедливо включение $G_{M} \cap E(M)$ $\subset T(M) \cap w A C(M)$, ав $X \in(R f) \cap(L U R)$ для $M \in \mathscr{F}(X)$ справедливо $G_{M}^{1} \subset T_{M}^{\prime}$ (см. также [2]). Последнее утверждение можно улучшить.

СлЕДСТвИЕ 2. Следующие условия эквивалентны:

a) $X \in(R f) \cap(L U R)$;

б) для любого $M \in \mathscr{F}(X) G_{M}^{1} \subset T_{M}^{\prime}$;

в) для любого $M \in \mathscr{F}_{\mathrm{cc}}(X) G_{M}^{1} \subset T_{M}^{\prime}$;

г) для любого $M \in \mathscr{F}(X) G_{M}^{1} \subset T(M) \cap w A C(M)$.

ДоказАтельство. а) $\Longrightarrow$ б). По предложению А в $X \in(R f)$ справедливо включение $G_{M}^{1} \subset \Gamma_{M}^{\prime}$. По определению $\Gamma_{M}^{\prime} \subset \delta^{\prime} S_{M}$. По теореме А в $X \in(L U R)$ имеем $\delta^{\prime} S_{M} \subset T_{M}^{\prime}$.

Импликации б) $\Longrightarrow$ в) и б) $\Longrightarrow$ г) очевидны.

в) $\Longrightarrow$ а), г) $\Longrightarrow$ а). Взяв в качестве $M$ замкнутое полупространство, получаем по теореме Джеймса $X \in(R f)$. По следствию $1 X \in(L U R)$ в случае в) $\Longrightarrow$ a). В случае г) $\Longrightarrow$ а) имеем $X \in(R f)$; следовательно, $X^{*}$ удовлетворяет условию $\left(w^{*} \omega C D^{*}\right)$. Тогда по теореме $1 X \in(w L U R)$, по теореме Б $X \in(C D)$, значит, $X \in(L U R)$.

СлЕДСТвИЕ 3. Следующие условия әквивалентны:

a) $X \in(R f) \cap(w L U R)$;

б) для любого $M \in \mathscr{F}_{\mathrm{cc}}(X) G_{M}^{1} \subset T(M) \cap w A C(M)$.

ДоказАтЕльство. а) $\Longrightarrow$ б). По предложению 1 из [11] в $X \in(R f)$ для любого множества $M \in \mathscr{F}_{\text {сс }}(X)$ справедливо включение $G_{M}^{1} \subset E(M)$. Тогда $G_{M}^{1} \subset G_{M} \cap E(M)$ и по следствию $1 G_{M} \cap E(M) \subset T(M) \cap w A C(M)$.

б) $\Longrightarrow$ а). Взяв в качестве $M$ полупространство и применив теорему Джеймса, получаем, что $X \in(R f)$, по следствию $1 X \in(w L U R)$.

\section{СПИСОК ЦИТИРОВАННОЙ ЛИТЕРАТУРЫ}

[1] Asplund E. Čebyšev sets in Hilbert space // Trans. Amer. Math. Soc. 1969. V. 144. P. 235-240.

[2] Fitzpatrick S. Metric projections and the differentiability of distance functions // Bull. Austral. Math. Soc. 1980. V. 22. № 2. P. 291-312.

[3] Fitzpatrick S. Differentiation of real-valued functions and continuity of metric projections // Proc. Amer. Math. Soc. 1984. V. 91. №4. P. 544-548. 
[4] Дудов С.И. Дифференцируемость по направлениям функции расстояния // Матем. сб. 1995. Т. 186. № 3. С. 29-52.

[5] Дудов С.И. Субдифференцируемость и функции расстояния // Матем. заметки. 1975. Т. 61 . № 4. С. $530-542$.

[6] Балаганский В. С. Дифференцируемость по Фреше функции расстояния и структура множества // Матем. заметки. 1988. Т. 44. №6. С. 725-734.

[7] Балаганский В.С.Достаточные условия дифференцируемости метрической функции // Тр. Института математики и механики. Т. 1. Екатеринбург: УрО РАН, 1992. С. 84-89.

[8] Балаганский В.С., Власов Л.П. Проблема выпуклости чебышевских множеств // УМH. 1996. Т. 51. №6(312). С. 125-188.

[9] Borwein J. M., Fitzpatrick S. P., Giles J. R. The differentiability of real functions on normed linear spaces using generalized subgradients // Math. Anal. Appl. 1987. V. 128. № 2 . P. 512-534.

[10] Zajíček L. Differentiability of the distance function and points of multi-valuedness of the metric projection in Banach space // Czech. Math. J. 1983. V. 33(108). P. 292-308.

[11] Балаганский В. С. Об аппроксимативных свойствах множеств с выпуклым дополнением // Матем. заметки. 1995. Т. 57. №1. С. 20-29.

Институт математики и механики УрО РАН, г. Екатеринбург 